\title{
On the on-site measurement of the degradation rate of crystalline silicon PV modules at plant level
}

\author{
Julio Pascual, Alberto Berrueta, Javier Marcos, Miguel García, Luis Marroyo \\ Department of Electric and Electronic Engineering and Institute of Smart Cities \\ Public University of Navarra \\ Pamplona, Spain \\ juliomaria.pascual@unavarra.es
}

\begin{abstract}
This paper proposes a method for measuring the degradation rate of crystalline silicon PV modules at plant level in two different ways as a form of verification. As actual levels of degradation rate have been observed to be as low as $0.2 \% /$ a, the uncertainties make it difficult to measure this value accurately at plant level. However, despite the low value, it is still important to know the actual degradation rate due to its impact on energy yield. In this paper, two ways of measuring the degradation rate at plant level are proposed. These two methods, with different uncertainty sources, are proposed to be used jointly in order to have a better approach to the real value. Finally, an example of measurement in a $1.78 \mathrm{MW}$ PV plant is presented.
\end{abstract}

Keywords-PV, degradation, measurement, crystalline silicon

\section{INTRODUCTION}

Global PV capacity is rapidly growing, with 40, 51 and 75 newly added GW in 2014, 2015 and 2016 respectively up to a cumulative PV installed capacity of $303 \mathrm{GW}$ [1]. According to [2], during 2017, PV capacity additions increased to $98.9 \mathrm{GW}$ totaling a cumulative PV installed capacity of $401.9 \mathrm{GW}$ globally by the end of 2017. This rapid growth is mostly due to cost reduction of PV modules down to $0.35 \$ / W$ in Q1 2017, bringing to an overall $\mathrm{PV}$ system cost of $1.03 \$ / \mathrm{W}$ and a levelized cost of energy in the U.S. between 0.04 and $0.06 \$ / \mathrm{kWh}$ (all data for utility-scale) [3]. Moreover, the cost reduction is being pushed down by the auction and tender systems used for procuring PV projects used nowadays in almost every big market [4]. In such a competitive market, it is critical for utilities to know the energy yield of their PV plants throughout their lifetime at the design stage, which, apart from the incident radiation on the PV modules and cell temperature, depends on the module's maximum power under standard test conditions $\left(P_{S T C}\right)$.

It is usually assumed that crystalline silicon PV modules suffer a degradation process, which lowers their $P_{S T C}$ linearly over their lifetime. This slow and continuous degradation occurs apart from other degradation mechanisms such as LID (which only occurs in the first hours), or common faults such as hot spots, micro-cracks, snail trails, etc. usually appearing in PV modules [5],[6] and which may affect more strongly to $P_{S T C}$. The degradation rate is usually guaranteed by the module manufacturer to be lower than $1 \% / \mathrm{a}(20 \%$ in 20 years) or $0.8 \% / \mathrm{a}$ ( $20 \%$ in 25 years). However, it is usually assumed that

This work has been supported by the Spanish State Research Agency (AEI) and FEDER-UE under grants DPI2016-80641-R and DPI2016-80642-R this degradation rate is around $0.5 \% / \mathrm{a}$ in accordance with analyzed cases by NREL and IEA up to 2012 [7],[8]. Moreover, recent studies suggest that this value may be closer to $0.2 \% / \mathrm{a}[9]-[12]$.

Despite the low value, it is still significant in terms of energy yield. For instance, the difference between assuming a degradation rate of $0.5 \% / \mathrm{a}$ or $0.2 \% / \mathrm{a}$ is a $3 \%$ of the energy yield of the PV plant during 20 years assuming a linear degradation, which is quite a significant value.

Measuring a $0.2 \% / \mathrm{a}$ degradation rate implies the measurement of $P_{S T C}$ in consecutive years with an accuracy very difficult to attain at plant level. The first measure in order to gain accuracy is to space measurements in time, but even after 4 years of measurements, it is still difficult to determine the degradation rate at plant level, as seen in [13]. However, after 10 years have passed since the first $P_{S T C}$ measurement, the expected difference to be observed is about $2 \%$, which requires a more attainable accuracy, yet with some difficulties, considering that during 10 years a PV plant may have suffered modifications. In addition, measuring devices' performance may have changed over time, even if calibrated. Moreover, after 10 years, measuring devices may have even been replaced by newer ones.

For this reason, in this paper, two methods for measuring the degradation rate at PV plant level with different uncertainty sources are proposed to be used jointly for the sake of redundancy and verification.

For the first method, maximum power of sampled parts of the plant is determined via I-V curves. These data can then be extrapolated to STC. Then, a method to extrapolate these data to the whole plant based on energy production and other parameters such as shading or downtime is applied [13],[14]. In this way, the whole PV plant $P_{S T C}$ can be estimated and compared to other years' measurements in order to calculate the degradation rate of PV modules. Measurements should cover as many years as possible in order to reduce uncertainty. The main uncertainty of this method comes from temperature measurements and extrapolation to STC.

Second method is based on the evaluation of the evolution of the yearly performance ratio $(P R)$ of the plant. This ratio gathers all inefficiencies of the PV plant. As such, $P R$ depends not only on $P_{S T C}$ but also on temperature, downtime due to 
equipment failure, soiling, etc. However, all parameters except $P_{S T C}$ and equipment failure are on average very similar from year to year and their variation can be assumed to be random. Moreover, during the first years energy loss due to equipment failure is very low, hence, the annual trend of $P R$ may well be assumed to be only caused by $P_{S T C}$ derating. However, if incremental energy loss due to equipment failure is noticeable and can be quantified, it should be taken into account. This second method has as main uncertainty sources measurement of energy production and radiation. One advantage of this second method is that data needed are usually readily available at PV plants.

Using these two methods with different uncertainty sources leads to a better understanding of the real degradation rate. These methods and a case study are presented in this paper. However, for a better appreciation of the degradation rate of crystalline silicon PV modules, a more extensive study should be carried out.

This paper is organized as follows. Section II explains both measuring methods in general terms. Section III shows in detail how to apply these methods to a particular PV plant and, finally, in Section IV the main conclusions are discussed.

\section{MEASURING METHODS}

\section{A. $P_{\text {STC }}$ evolution}

This first method consists on measuring $P_{S T C}$ of the plant on a yearly basis, spacing first and last measurements as much as possible; recommended time span is at least 10 years. The yearly trend of $P_{S T C}$ is the degradation rate. Note that this method relies on several $P_{S T C}$ measurements, and does not rely on nameplate $P_{S T C}$, which, in some cases, as in the case study, has been observed to be quite above real $P_{S T C}$ at the time of installation of the plant.

For this method, the plant is split in the smallest sections for which energy yield is known. For every section, the differences that would make them have a different energy yield such as different shading or downtime periods are evaluated. In this way, every section energy yield can be normalized so that $P_{S T C}$ is the only variable affecting normalized energy yield. Moreover, $P_{S T C}$ and normalized energy yield can be considered directly proportional.

The methods for normalizing energy yield will depend on the plant configuration; however, provided that all PV modules are the same model and are equally oriented it can be assumed that they are all equally affected by temperature and soiling. Another general assumption, when inverters are all the same model, is that inverter and $\mathrm{AC}$ losses are equal for all sections of the plant. Considering this, shading and downtime are the main variables to be considered for normalization. The method for normalizing will be explained in more detail in Section III, for the case study.

Secondly, few sections with a representative normalized energy yield (i.e., avoiding the sections of the PV plant with the highest and lowest normalized energy yields) are selected for measuring their maximum power under real conditions $\left(P_{M}\right)$ with an I-V tracer. In addition, irradiance $(G)$ and cell temperature $\left(T_{C}\right)$ are simultaneously measured. The techniques used for measuring $G$ and $T_{C}$ might depend on the PV plant configuration (available mounted sensors, accessibility of PV modules, etc.); the technique used in the case study will be explained in Section III. With these data, maximum power of measured sections can be extrapolated to STC using the following two-parameter model of a PV module:

$$
P_{S T C}=P_{M} \cdot \frac{1000}{G} \cdot \frac{1}{\left[1+\gamma \cdot\left(T_{C}-25\right)\right]}
$$

where $T_{C}$ is in ${ }^{\circ} \mathrm{C}, G$ in $\mathrm{W} / \mathrm{m}^{2}$ and $\gamma$ is cell maximum power temperature coefficient, usually given by the manufacturer and usually around $-0.45 \% /{ }^{\circ} \mathrm{C}$ for c-Si cells. This method is widely accepted for its combination of both simplicity and accuracy [15],[16]. However, when measuring large areas (i.e. $500 \mathrm{~kW}-$ $1 \mathrm{MW})$ it is sometimes seen as a better solution to use $P_{M P P}$ records, although, I-V curves still present similar results if special care is taken when measuring $G$ and specially $T_{C}$ [17],[18]. Also note that, as I-V tracing is an instantaneous measure, in order to compare results with other years, soiling should be accounted for by measuring the maximum power of sampled modules before and after cleaning them.

After having obtained $P_{S T C}$ of selected parts of the plant, and having normalized every section in terms of energy yield, $P_{S T C}$ can be calculated for each section assuming that normalized energy yield and $P_{S T C}$ are directly proportional. Finally, the whole plant $P_{S T C}$ is calculated as the weighted average of the $P_{S T C}$ of every section. Applying this method for several years, the yearly variation of $P_{S T C}$ over time can be calculated, which is in fact the PV modules' degradation rate.

The main uncertainty of this method is the extrapolation to STC. Temperature measurement is particularly critical and its measurement is difficult for large generators, the main reason being that its value is not uniform along the generator, especially as wind speed increases. For this reason, test conditions should be carefully taken into account. In particular [18] recommends an I-V capture time of above $20 \mathrm{~ms}$, incident irradiance larger than $800 \mathrm{~W} / \mathrm{m}^{2}$, diffuse/global irradiance proportion lower than $20 \%$, and wind speed lower than $3 \mathrm{~m} / \mathrm{s}$. In addition, the value of $\gamma$ given by the manufacturer has been shown to present discrepancies with measured values [19], hence, $T_{C}$ should be as close to STC as possible. In this way, the propagation error due to a wrong value of $\gamma$ would be drastically reduced, ideally resulting in a zero error in the case of measuring at $T_{C}$ of $25^{\circ} \mathrm{C}$.

\section{B. Yearly PR evolution}

This second method relies on data usually available at PV plants, namely, radiation on the plane of PV modules and energy yield. However, radiation is sometimes measured in the horizontal plane. If that is the case, radiation needs to be transformed to the plane of the modules. The particular method used for the case study can be found in Section III. Yearly $P R$ can then be computed as: 


$$
P R=\frac{E_{a} / P_{S T C, n}}{H_{a} / G_{r e f}}
$$

where $E_{a}$ is the energy yield of the PV plant during one year, $H_{a}$ is the annual radiation over the plane of the PV modules and $G_{r e f}$ is the irradiance at STC, i.e. $1000 \mathrm{~W} / \mathrm{m}^{2}$. By doing this on a yearly basis, the trend of the PV plant's $P R$ can then be obtained.

$P R$ is affected by many factors apart from actual $P_{S T C}$, such as shadows, soiling, temperature, etc. However, on a yearly basis, all factors except $P_{S T C}$ remain almost constant, or rather, vary randomly, hence, the yearly evolution of $P R$ can be seen as the yearly evolution of $P_{S T C}$, i.e., the degradation rate. One exception to this may be PV plant's downtime due to maintenance, which may slightly increase over time as the plant ages. If this effect is noticeable and can be quantified, it should be taken into account.

One of the most important sources of uncertainty of this method is the transformation of horizontal radiation to the inclined plane so, when possible, radiation should be measured in the plane of the PV modules. Regarding energy yield is usually measured accurately by the meters used for billing. Finally, radiation is usually measured accurately; however, in such long periods, data points are sometimes missing. Nearby weather stations may be used to fill the gaps or for crosschecking.

\section{CASE STUDY}

As an example, in this section, the degradation rate measuring methods are applied to a $1.78 \mathrm{MW}$ PV plant in northern Spain. The PV plant comprises 280 single-axis azimuth-tracking systems, hereinafter referred to as generators. The generators, with a nameplate $P_{S T C}$ of $6.36 \mathrm{~kW}$ are arranged in a square grid, as shown in Fig. 1. Regarding power meters, most generators have their own unit, however, some grouped generators share one meter. In total, there are 199 power meters in the PV plant. Energy data are available on a monthly basis.

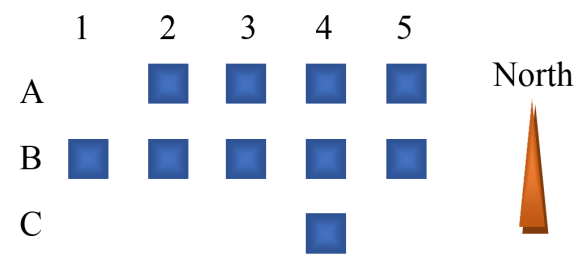

Fig. 1. Detail of 10 generators (squares) in the PV plant. Spacing between generators in this plant is $16 \mathrm{~m}$.

\section{A. $P_{\text {STC }}$ evolution}

First step is to normalize the energy yield of the PV plant's smallest sections; in this case, the smallest section that can be analyzed is the generator.

Generators shade each other differently depending on their position with respect to each other. For instance, generator $4 \mathrm{C}$ in Fig. 1. receives no shading. However, generator $2 \mathrm{~B}$ is shaded by generators on both sides (1B and $3 \mathrm{~B}$ ). Generator $4 \mathrm{~B}$ is shaded just like $2 \mathrm{~B}$, because generators in this plant never cast a shadow over the generator just northward. However, generator $3 \mathrm{~A}$ is shaded not only by side generators $(2 \mathrm{~A}$ and $4 \mathrm{~A}$ ) but also by generators $2 \mathrm{~B}$ and $4 \mathrm{~B}$ but not $3 \mathrm{~B}$ as explained before. In general, seven groups of generators have been differentiated according to their energy loss due to shading. For every group, the median of the monthly production is chosen as the reference value, and the monthly energy yield of every generator can be normalized with respect to its own shading group.

Generators' downtime, usually due to failure of the inverter or the tracking system, although rarely occurring (less than $1 \%$ of energy yield is lost for this cause in the case study) is also accounted for. To do so, whenever the normalized energy yield drops significantly and recovers the next month, it is assumed to be caused by a temporary failure and not due to a degradation process of the PV module. Accordingly, these few data points are removed for further calculations.

Having normalized energy yield, only few generators' $P_{S T C}$ need to be measured in order to estimate the plant's $P_{S T C}$. In this case, six generators were chosen for $P_{S T C}$ measuring. For this purpose, irradiance and $T_{C}$ need to be measured. Irradiance is measured with a reference PV module as proposed in [17], via its short-circuit current. Regarding $T_{C}$, different techniques might be used; in this case, an infrared camera is used to measure several points of the backsheet of the PV modules. $T_{C}$ is obtained by computing the average of these measurements. $P_{S T C}$ is then calculated and plotted against normalized energy yield.

In order to estimate the rest of the generators' $P_{S T C}$, a linear regression, forced to pass through $(0,0)$, is carried out between normalized energy yield and $P_{S T C}$ (Fig.2.). Following the resulting linear equation, $P_{S T C}$ can then be estimated for every generator according to their normalized energy yield.

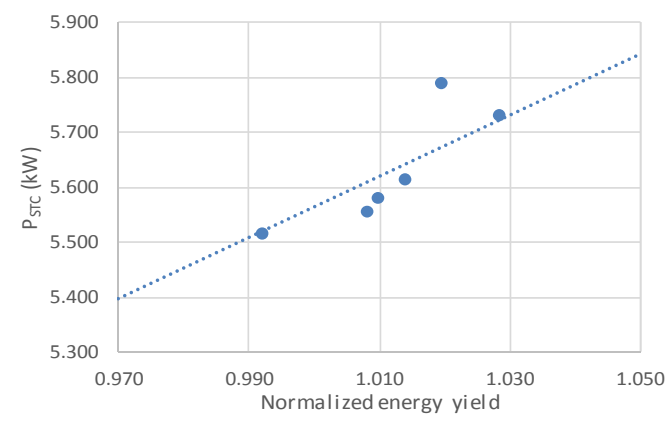

Fig. 2. Linear regression of normalized energy yield and measured $P_{S T C}$.

Finally, the whole plant $P_{S T C}$ can be calculated as the weighted average of the $P_{S T C}$ of every generator. In this case, the average $P_{S T C}$ value is $5567 \mathrm{~W}$ or 0.875 of the nameplate $P_{S T C}$ power.

Comparing the value obtained with previous years' analysis, degradation rate can now be calculated. As shown in Fig. 3, a linear regression suggests that degradation rate is $0.40 \% /$ a. Note that this PV plant was installed in 2005, hence, even though $P_{S T C}$ values are very low in 2016, degradation is not the main cause. The cause is an abnormal low initial $P_{S T C}$, which is out of the scope of this paper. Worst and best cases 
are $0.49 \% / \mathrm{a}$ and $0.36 \% / \mathrm{a}$ respectively. Among these values, $0.49 \% / \mathrm{a}$ is, in principle, the value with the highest uncertainty, as it only uses two points (years 2009 and 2016), separated by 7 years. On the other hand, the value $0.36 \% / \mathrm{a}$, despite being also obtained with just two points (2006 and 2016), these are separated by 10 years, resulting in a lower relative uncertainty.

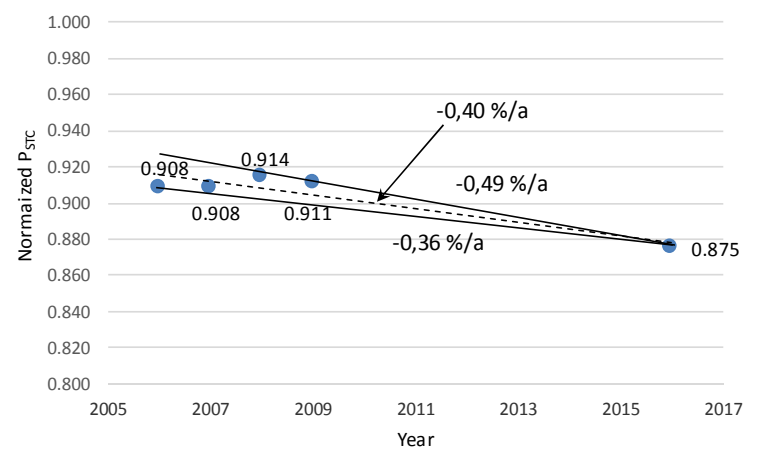

Fig. 3. Linear degradation rate according to campaings in 2006, 2007, 2008, 2009 and 2016. Degradation rate lies between $0.36 \%$ a and $0.49 \% / \mathrm{a}$.

In this case study, it can be appreciated that the measured value is different depending on the years used. By measuring $P_{S T C}$ every year, a more representative value is obtained, yet quite a wide range of values is obtained. The second method helps in the interpretation of these values.

\section{B. Yearly PR evolution}

For this method, firstly, yearly production (in $\mathrm{kWh}$ ) is divided by nameplate STC power (in $\mathrm{kW}$ ). Next, yearly radiation on the plane of the modules is needed. In the case study, only horizontal radiation was available, but on a monthly basis. This made it possible to convert monthly horizontal radiation into radiation on the plane of the modules as in [20]. This is an approximation and represents the biggest source of uncertainty of this second method.

With these two sets of data, last step is to calculate $P R$ using (2). In this case, as data for every year of study exist, and there is no apparent anomalies in the data (i.e., no apparent outliers) only linear regression is considered. Assuming that evolution of $P R$ is mainly due to degradation of $P_{S T C}$, it can be said that degradation rate according to the second method is $0.23 \% / \mathrm{a}$, as shown in Fig. 4 .

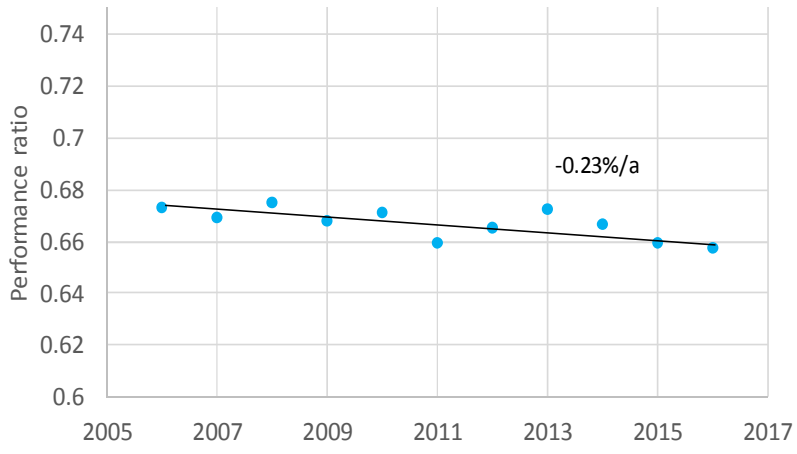

Fig. 4. $P R$ graph shows a negative evolution of $0.23 \% / \mathrm{a}$.
Note that, as real $P R$ may include other incremental energy losses than those only due to PV module degradation, its trend at least represents an upper bound to the real degradation rate. As such, a lower measured value of the $P R$ trend than the degradation rate obtained in the first method (which might happen due to uncertainty, and in fact happens in the case study) suggests that measured degradation rate in the first method must have been biased towards greater values so that real value must be lower.

\section{CONCLUSIONS}

This paper addresses the problem of measuring the degradation rate of crystalline silicon PV modules at plant level. In this paper, two methods are presented for the measurement of the degradation rate of crystalline silicon PV modules at plant level. First method is based on the direct measurement of maximum power, irradiance and cell temperature. Extrapolation to STC and comparison with other years' measurements reveals a first approach to the degradation rate. This method is particularly sensitive to cell temperature measurement for which special care must be taken. Given that degradation rate can be as low as $0.2 \% / \mathrm{a}$, it is recommended that about 10 years separate first and last measurements. This, however, leads to other uncertainty sources, such as equipment and plant modifications over time. As such, this method reveals a wide range of degradation rate values depending on the analyzed year. For this reason, in order to crosscheck the values obtained with the first method, a second method with different uncertainty sources is proposed. The second method is based on energy yield and incident radiation measurements, which are variables usually available at PV plants. This second point of view provides new information and, for instance, in the case study, suggests that the degradation rate is not as high as observed with the first method. Note that, although using these two methods gives a better understanding of the degradation rate than using just one of them, uncertainty is still high when measuring just one plant. For a more accurate study on the actual value of the degradation rate of crystalline silicon PV modules, a more extensive measuring campaign should be carried out. However, this study already shows that degradation rates are well below guaranteed values; moreover, it suggests that degradation rates might be even below the $0.5 \%$ /a generally accepted.

\section{REFERENCES}

[1] REN21, "Renewables 2017. Global status report", ISBN 978-39818107-6-9

[2] Solar Power Europe, "Global Solar Market Grows Over 29\% in 2017 with even more to come in 2018", 2018.

[3] R. Fu, D. Feldman, and R. Margolis, "U.S. Solar Photovoltaic System Cost Benchmark Q1 2017", National Renewable Energy Laboratory (NREL), 2017.

[4] B. Attia, M. Parikh, T. Heggarty, "Global Solar Demand Monitor: Q1 2017. Market trends update", GTM research, 2017.

[5] A. Dolara, G. C. Lazaroiu, S. Leva, G. Manzolini and L. Votta, "Snail Trails and Cell Microcrack Impact on PV Module Maximum Power and Energy Production," in IEEE Journal of Photovoltaics, vol. 6, no. 5, pp. 1269-1277, Sept. 2016.

[6] F. Grimaccia, S. Leva, A. Dolara and M. Aghaei, "Survey on PV Modules' Common Faults After an O\&M Flight Extensive Campaign 
Over Different Plants in Italy," in IEEE Journal of Photovoltaics, vol. 7, no. 3, pp. 810-816, 2017.

[7] Report IEA-PVPS T13-08:2017, “Technical Assumptions Used in PV Financial Models. Review of Current Practices and Recommendations", May 2017.

[8] D.C. Jordan and S.R. Kurtz, "Photovoltaic degradation rates-an analytical review," Prog. Photovolt. Res. Appl., vol. 21, no. 1, pp. 1229, Jun. 2012

[9] K. Kiefer, D. Dirnberger, B. Müller, W. Heydenreich, A. Kröger-Vodde, "A degradation analysis of PV power plants", 25 ${ }^{\text {th }}$ PVSEC, 2010, Valencia (Spain)

[10] M. Alonso-Abella, F. Chenlo, A. Alonso and D. Gonzalez, "Toledo PV plant 1MWp - 20 years of operation”, 29th EU PVSEC, 2014, Amsterdam.

[11] A. Pozza, T. Sample, "Crystalline silicon PV module degradation after 20 years of field exposure studied by electrical tests, electroluminescence, and LBIC", Prog. Photovolt: Res. Appl. vol. 24, pp. 368-378, 2016.

[12] T. Ishii, A. Masuda, "Annual degradation rates of recent crystalline silicon photovoltaic modules", Prog. Photovolt: Res. Appl., vol. 25, pp. 953-967, 2017.

[13] M. García, L. Marroyo, E. Lorenzo, J. Marcos and M. Pérez, "STC power for $15 \mathrm{MW}$ of PV comparing nameplate, initial power and power after 4 years”. Prog. Photovolt: Res. Appl., vol. 22, pp. 242-247, 2014.

[14] M. García, J. A.Vera, L. Marroyo, E. Lorenzo and M. Pérez, "Solar-tracking PV plants in Navarra: A $10 \mathrm{MW}$ assessment", Prog. Photovolt: Res. Appl., vol. 17, pp. 337-346, 2009.

[15] M. Fuentes, G. Nofuentes, J. Aguilera, D.L. Talavera, M. Castro, "Application and validation of algebraic methods to predict the behaviour of crystalline silicon PV modules in Mediterranean climates", Solar Energy, vol. 81, pp. 1396-1408, 2007.

[16] C. R. Osterwald, "Translation of device performance measurements to reference conditions", Solar cells, vol. 18, pp. 269-279, 1986.

[17] I. de la Parra, M. Muñoz, E. Lorenzo, M. García, J. Marcos, F. MartínezMoreno, "PV performance modelling: A review in the light of quality assurance for large PV plants", Renewable and Sustainable Energy Reviews, vol. 78, pp 780-797, 2017.

[18] R. Moretón, E. Lorenzo and J. Muñoz, “A 500-kW PV generator I-V curve", Prog Photovolt 2013.

[19] G. Makrides, B. Zinsser, M. Norton, G.E. Georghiou, M. Schubert, J.H. Werner, "Error sources in outdoor performance evaluation of photovoltaic systems", 24th EU PVSEC, pp 3904-3909, Hamburg, Germany, 2009.

[20] E. Lorenzo, "Energy collected and delivered by PV modules" in Handbook of photovoltaic science and engineering, Edition 1, A. Luque, S. Hegedus, Ed. Chichester, England: Wiley, 2003, pp. 905-970. 